\title{
Composite inhibitor for gas hydrate in deepwater drilling
}

\author{
Xiaolan Liu ${ }^{1, a}$, Xianghua Liü,b and Wenqian Wei ${ }^{3, c}$
}

${ }^{1}$ Drilling Technology Research Institute of Shengli Petroleum Engineering Corporation Limited, Sinopec

\author{
${ }^{2}$ Yellow River Drilling Corporation of Shengli Petroleum Administration Bureau,Sinopec \\ ${ }^{3}$ China University of Petroleum (Hua Dong) College of Petroleum Engineering Shandong Qingdao \\ a151816360@qq.com, ${ }^{b}$ liuxianghua708.slyt@sinopec.com, ${ }^{c}$ 348437190@qq.com
}

\begin{abstract}
Keywords: Natural gas hydrate, inhibitor, composite inhibitor, preventing hydrate formation Abstract. In recent years, the research about natural gas hydrate inhibitors has transformed from thermodynamics inhibitors to kinetic inhibitors and anti-agglomerant inhibitors in the deepwater drilling. However, once the malfunction occurred in the injection system, kinetic inhibitors is powerless facing the hydrate plug resulted from irregular shut-in gas wells or lack of inhibitors. Meanwhile, surfactants would not be economically viable as a single inhibitor. Considering the single inhibitors have some limitations in the practical application, kinetic inhibitors, anti-agglomerant inhibitors, thermodynamic inhibitors and other auxiliary agent are used in combination to prevent hydrate plug. This article is a comprehensive study of the advantages and disadvantages of various inhibitors, which develops a complex scheme, reduces the amount of compsite inhibitors, increasing the supercooling degree, and will be more suitable for industrial application.
\end{abstract}

\section{Introduction}

In deepwater drilling, natural gas hydrate is easy formate in the choke line, anti BOP and subsea wellhead and other parts in the drilling of shallow gas, due to the low temperature condition of submarine. The clathrate compound once coalescence together, can plug the pipeline and bring security risks for oil and gas development safety.

In order to inhibit the formation of hydrate, the domestic and foreign scholars continue to explore the formation mechanism, distribution and prevention technology of natural gas hydrate. Foreign experts and scholars have shown that the most widely used method of treatment is to add chemical additives in drilling fluid. In the early stage, the thermodynamic inhibitors such as methanol, ethylene glycol, etc., are used to change the equilibrium conditions of the hydrate. This kind of reagent is added in a large amount, the general as high as 30 40\%, and some even reached the weight ratio of $50 \%$, high cost, but may also exist in the pipeline corrosion problems, the individual will pollute the environment.In recent years the scientists begin to study with low dose and low cost new kinetic inhibitor to replace the thermodynamic inhibitors. Kinetic inhibitor is the extension of hydrate nucleation induction time or changing the crystal aggregation process to delay the hydrate crystal growth and aggregation, so as to achieve the effect of inhibiting the formation of gas hydrate, which has been through the experiment confirmed. Through experimental study, the effect of kinetic inhibitors is to delay the formation of hydrate effectively, and little amount, good effect, and the cost is not high, little pollution to the environment.

Once hydrate has been formated, the kinetic inhibitor could not eliminate the hydrate. Therefore, this thesis has carried out a series of research work on compound inhibitors. By the experimental verification of the hydrate thermodynamic inhibitors, kinetic inhibitors and surface active agent anti agglomerant compound test is carried out, to play a synergistic effect of the coordination of a variety of inhibitors and the mixed reagents, carry out hydrate inhibition performance experiments, then develop a new composite hydrate inhibitor. 


\section{Kinetic inhibitors and inorganic salt inhibitors}

Polyacrylamide (PAM) and low viscosity carboxymethyl cellulose slurry filter loss agent( lv-cmc), which have been commonly proven capable of inhibiting the formation of gas hydrate in drilling fluid additives, will be the two kinetic inhibitor and inorganic salt thermodynamic inhibitors mixed.

The experimental conditions were carried out under the salt water system with a concentration of $5 \%$. Figure 1 is a PAM composite inhibitor system with 5\% inorganic salt, and figure 2 is a LV-CMC complex inhibitor system with 5\% inorganic salt

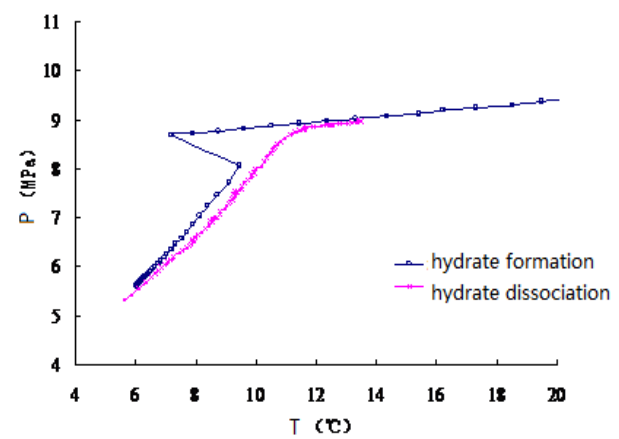

Fig. 1 PAM composite inhibitor system with 5\% inorganic salt

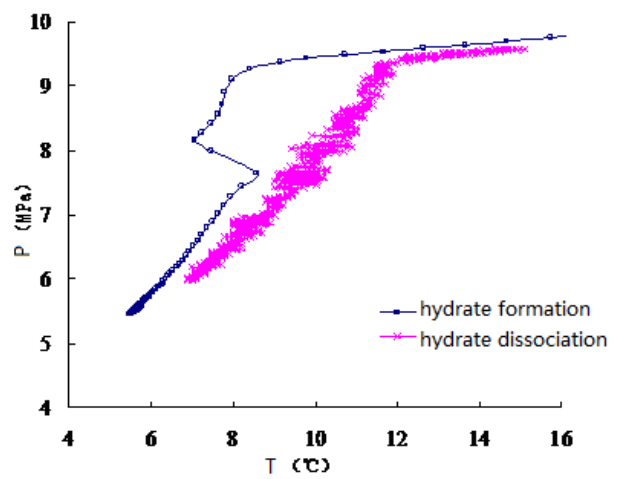

Fig. 2 LV-CMC complex inhibitor system with $5 \%$ inorganic salt

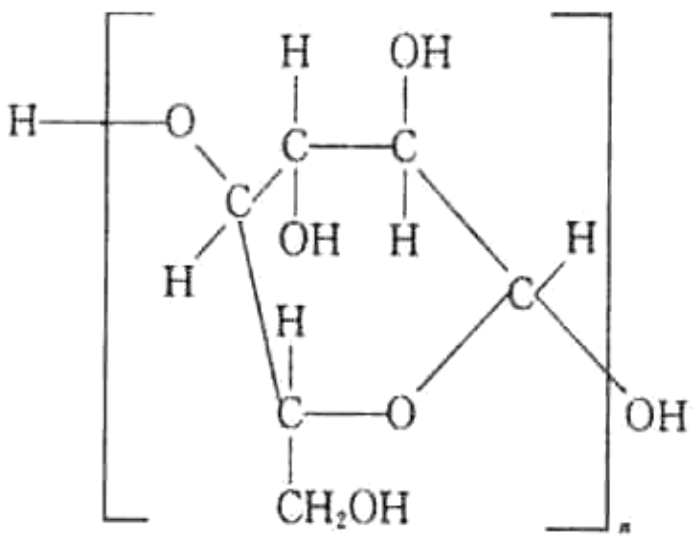

Fig. 3 LV-CMC structure

Hydrate formation and stay stable system pressure of hydrate dissociation curves are shown in Figure 1 .The corresponding phase state with pressure and temperature respectively is 11.658 centigrade and $8.848 \mathrm{MPa}$. Using the Sloan forecast software, the degree of over cooling was 0.537 . It shows that the hybrid system has a certain ability to inhibit hydrate formation. Analysis of the formation of hydrates, the main reasons of the delay phenomenon is the non nuclear power groups of water molecules produced a kind of interaction., disrupt the liquid water molecules through hydrogen bonding generated grid, making water molecules form a grid to overcome the interaction, so as to delay the effects of hydrate formation.

The degree of over cooling was 1.023 in fig. 2 by the same way. Therefore, the mixed system has the effect of inhibiting the formation of hydrate. Lv-cmc (Fig. 3)has high molecular link hydroxyl and ether oxygen radical active groups absorbed into the hydrate crystal surfaces, polymer forcing 
hydrate crystals with small curvature radius around the polymer or polymer link growth. Therefore, it can reduce hydrate formation rate and extension of hydrate nucleation induction time or change the crystal aggregation process.

\section{Kinetic inhibitors and the combination of alcohol inhibitors}

Methanol and ethylene glycol are used commonly as thermodynamic inhibitors. As methanol has a certain toxicity, it is not conducive to the operation of the laboratory, so the liquid is mass of $40 \%$ volume ratio ternary caprolactam polymers and $10 \%$ ethylene glycol water solution. Based on this composite, the inhibition performance of the compound inhibition system was evaluated. Experimental results (Fig. 4) show that composite inhibitor system also has the property of hydrate inhibition, and the composite inhibitor system nearly delay 50 hours than ethylene glycol as inhibitor in hydrate formation, so the composite system delay hydrate formation effect is obvious. Using ethylene glycol has changed the hydrate formation conditions for nucleation from the point of view of thermodynamics, and after mixed with the ternary caprolactam later, add new ternary caprolactam polymer as a kinetic inhibitor for drilling fluid, it can inhibit the growth rate. Two types of common inhibitors effect together, then hydrate formation is delayed.

\section{Kinetic inhibitors and common surfactants for drilling fluids}

Mix caprolactam polymer dynamics inhibitor and Spann series surfactants. The fluid allocation of mass to volume ratio is $40 \%$ of the drilling fluid with ternary caprolactam polymer aqueous solution and $0.1 \%$ span series of surface active agent as a composite inhibitor system. First, the mass volume ratio of $10 \%$ of the polymer - surfactant - water - methane system, under different pressures, the mixed solution was tested. The graphics of the compound hydrate inhibitor system were compared, and the results are shown in Figure 5.

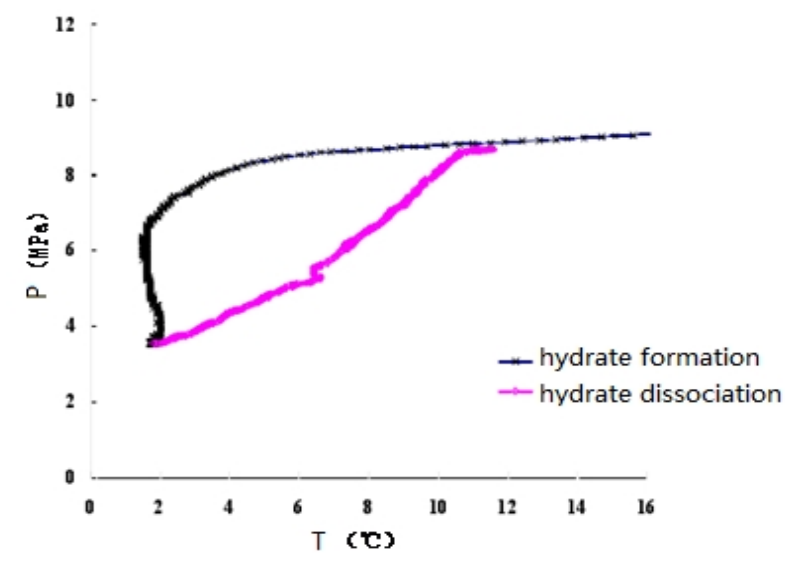

Fig. 4 Caprolactam and ethylene glycol compound system hydrate inhibitor

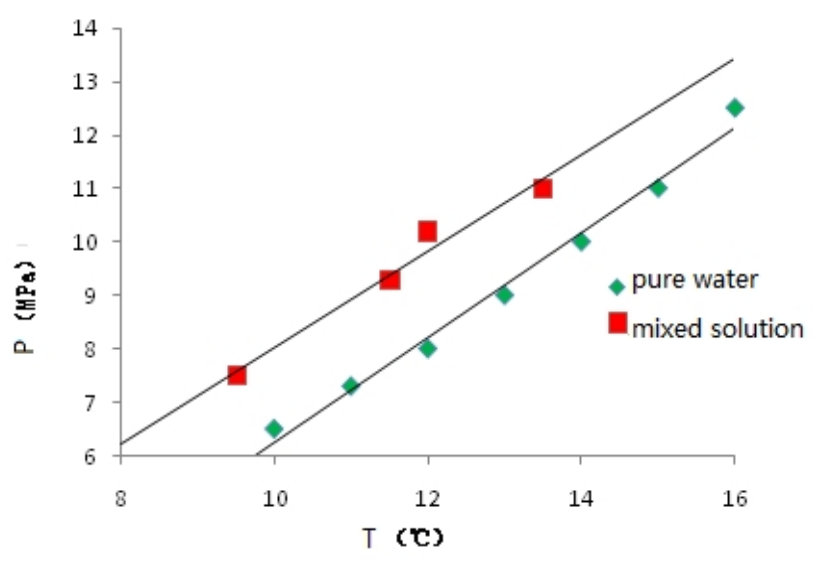

Fig. 5 compound hydrate inhibitor compared with pure water

The polymer kinetic inhibitors, containing the main role of the lactam ring, is good to adsorb on the hydrate surface, through eutectic or adsorption, entering the hydrate cage shaped cavity and with the surface of hydrate hydrogen bond formation, which adsorbed on the surface of hydrate, the hydrate with small radius of curvature around, or generated between the polymer chains; also from the space so as to prevent the gas into hydrate cavities, prevent the growth of hydrate nucleation, or hydrate particle retention to disperse, thereby inhibiting the formation of hydrates. While the active 
agents prevent the aggregation of hydrate grains by adsorption or emulsification, so that the hydrate crystal can be a dispersed state in the temperature which is not very low.So as to delay or inhibit the formation of hydrate.

\section{Summary}

(1) the experiments study preliminary kinetic inhibitors and inorganic salts thermodynamic inhibitors, ethylene glycol and surface active agent compounded, through the measurement of hydrate formation time and cooling degree respectively, different composite inhibitor system performance of hydrate inhibition was evaluated, get a new hydrate inhibitor, and verify the synergistic effect of the coordination of composite inhibitors.

(2) in addition to the above research, we can also carry out the experimental exploration of a variety of other combinations. At present, there are many problems, such as the lack of practical application experience, the combination of experiment and practical application, more new inhibitors still need to be further studied.

\section{References}

[1] Heidaryan E, Salarabadi A, Moghadasi J, et al. A New High Performance Gas Hydrate Inhibitor[J]. J Nat Gas Chem, 2010, 19(3): 323 - 326

[2] Cohen J M, Wolf P F, Young W D. Enhanced Hydrate Inhibitors: Powerful Synergism with Glycol Ethers[J]. Energy Fuels, 1998, 12(2): 216 - 218

[3] Kelland M A, Iversen J E. Kinetic Hydrate Inhibition at Pressures up to 760 bar in Deep Water Drilling Fluids[J]. Energy Fuels, 2010, 24(5):3003 - 3013

[4] Hu Jun, Li Sijia, Wang Yanhong, et al. Kinetic Hydrate Inhibitor Performance of New Copolymer Poly (N-Vinyl-2-Pyrrolidone-co-2-Vinyl Pyridine)s with TBAB[J]. J Nat Gas Chem, 2012, 21(2):126 - 131

[5] Cheng Chua Pei, Kelland M A. Tetra(iso-Hexyl)Ammonium Bromide: The Most Powerful Quaternary Ammonium-Based Tetrahydrofuran Crystal Growth Inhibitor and Synergist with Polyvinylcaprolactam Kinetic Gas Hydrate Inhibitor[J]. Energy Fuels, 2012, 26(2): 1160 - 1168

[6] Richard A R, Adidharma H. The Performance of Ionic Liquids and Their Mixtures in Inhibiting Methane Hydrate Formation[J]. Chem Eng Sci, 2013, 87(3): 270 - 276 